\title{
How Sweet It Is: Sugar Consumption and its Implications for Human Resource Management and the Work-Life Divide
}

\author{
Gareth Craze \\ Case Western Reserve University
}

\begin{abstract}
Consumption of refined sugar in modern diets has been identified as a source of many modern illnesses, including emotional and mood disorders. Furthermore, workplace "cake culture" within organizations has institutionalized and ritualized the consumption of sugar. I outline the effect that sugar consumption has on stress, fatigue and mood, and propose that these effects will have deleterious consequences on individual-level health and wellbeing in organizations. I further propose that social contagion has a mediating effect on these interactions. Implications for human resource management (HRM) and the work-life divide are then discussed. The paper concludes with future research directions for scholars.
\end{abstract}

\section{INTRODUCTION}

A sizeable literature (see e.g. Johnson, Sánchez-Lozada, Andrews, \& Lanaspa, 2017; Taubes, 2016) has begun to emerge - both in scholarly research across a number of disciplines, and in popular science writing and journalism - that has identified refined sugar consumption as a primary cause of many modern maladies. There is now increasingly vocal convergence on the proposition that sugar has had some central causal role in propagating a host of so-called "diseases of civilization"; diseases whose rate of incidence increases in a population relative to increasing degrees of affluence and resource affordability. As recently as the Industrial Revolution, sugar was exclusively an indulgence of the very wealthy. Refinements in sugar cane production and the advent of sugar beet production in Europe in the last few centuries, coupled with the sustained industrialization and commercialization of the sugar industry, has now led to a zeitgeist in which the average American adult consumes 5-6 times the recommended daily intake of sugar laid out by the World Health Organization's recently revised global nutritional guidelines (World Health Organization, 2015). The sheer proliferation of industriallyprocessed foods loaded with refined sugar, and the consumption thereof, within even only the most recent three decades, represents arguably the most impactful shift in human dietary patterns since the Agricultural Revolution some 10-12 millenia ago.

Chief among the "diseases of civilization" aforementioned are conditions such as obesity and Type 2 diabetes, which have long been at or approaching epidemic levels in most Western (and thus most affluent) cultural contexts, but which also include emotional and mood disorders such as depression. While once thought to be little worse than merely a source of "empty calories", sugar has been increasingly recognized as having distinct micro-nutrient properties which are capable of compromising healthy cognitive and affective functioning, including noted effects on learning and memory, as well as emotion and mood regulation. Indeed, the effects of sugar on the human body are now thought by a 
number of scholars to share many of the same metabolic pathways as corporeal illnesses such as obesity and diabetes (Carrera-Baston, Fontes-Villalba, O'Keefe, Lindeberg \& Cordain, 2011; Mansur, Brietzke \& McIntyre, 2015).

The consumption of refined sugar has been demonstrated to have significant effects on stress, fatigue and mood. These considerations may be of significant import for organizations, and for human resource management practitioners more specifically. What has been referred to as "cake culture" - the consumption of sugar-rich foods as a prominent element of much modern office-based organizational life - might serve as a vector for the transmission of chronic stress, fatigue and negative mood among organizational members through the medium of social contagion. In the present paper, I propose that sugar's uniquely deleterious effects on organizationally-relevant cognitive and affective phenomena (namely, though not limited to, stress, fatigue and mood) poses practical and ethical concerns for human resource management practitioners.

Specifically, I present the case that, insomuch as sugar can produce deleterious individual-level mental health effects, these effects can be extended to the organizational collective through social contagion, and then amplified in the context of organizations where cake culture is prevalent. I additionally pose the notion that these considerations have significant implications for human resource practice with respect to respecting an individual's work-life balance (or work life/private life divide), and whether sugar consumption in the present day might come to be identified in a similar light, with respect to human resource policy and practice, as tobacco smoking has become in recent decades. I begin with an overview of both the work-life divide in the context of human resource management and the nature of cake culture in modern organization life. I then identify the deleterious effects that consumption of sugar produces on stress, fatigue and mood (and how each of these in turn has effects on sugar consumption), and make several propositions accordingly. A mediating effect of social contagion on these effects is then proposed. The paper closes with practical and ethical implications for practitioners and recommendations for future research efforts, including a call for focused interdisciplinary efforts.

\section{MANAGING THE WORK-LIFE DIVIDE}

Helping to provide the conditions under which an employee can strike a balance between work and life, and navigate the associated divide between their private life (broadly encompassing any of their activities and engagements outside the work sphere) and work life, has long been an important consideration in the organizational and managerial sciences generally, and studies of human resource management (HRM) specifically. In an age where it is common for employees to take work home with them, remain in communication with their organization even while on vacation, and otherwise put in work hours that they would otherwise use to recreational or rejuvenating ends, this consideration has become an increasing source of vigilance and duty of care for HRM practitioners. Exacerbating matters still, upticks in remote employment and telework have further blurred the lines between the office and the home; in turn boosting the necessity of HRM oversight in ensuring work-life balance for employees (Kramar, 2014).

Indeed, with chronic stress, fatigue and burnout rates in organizations continuing to skyrocket, and associated increases in absenteeism and turnover and declines in productivity continuing to befall many organizations across a number of industries, managing employee energy, emotions and moods ought to be of premium concern for any proactive human resources department that values employee wellness and performance in equal measure. The entitlement to, and sanctity of, a private life that is not unnecessarily interfered with on account of vocational demands and spillover, is one of the foremost recognitions HRM practitioners can extend to employees in the service of optimizing their general wellbeing as a person (Maslach, Leiter \& Jackson, 2012).

Inherent in the proposition that there is a functional and necessary divide between the realms of private and work life is the notion that there are boundaries which define an individual's relationship to their organization and which contextualize the performative and interactive dimensions of that relationship. These boundaries can span multiple axes, and needn't be as straightforwardly binary as the 
common work-life terminology would suggest. Particularly in light of the aforementioned developments in the nature of the work-life divide, there are often extra-organizational and extra-individual influences which reconfigure or obscure the boundaries between work life and private life. Consider the act of cognition. As much as many an employee would like to think otherwise, they do not leave the office with a different brain to the one they walk out the front door with in the morning. The multiple, often overlapping demands of everyday life are such that the cognitive demands of both home and work life will influence each other to some degree in all but the most ideal of cases (Ernst Kossek, Lewis \& Hammer, 2010).

Good HRM practice in this area walks a practical and ethical tightrope between on one hand being sensitive to the extra-work influences of an employee's private life and how these might impact upon job performance, and on the other hand honoring the demands of the organization and ensuring that employees are sufficiently responsible and conscientious so as to manage the pressures of their private life in such a way that the organization, and their role within it, is not unduly affected or disadvantaged.

\section{"CAKE CULTURE"}

The sharing and consumption of food is an enduring feature of work life in many organizational settings. As a source of ritual and social lubrication, workplace gatherings centered on collective eating is as embedded an extra-work institution as any one will find across a number of organizations and industries. Indeed, in some organizations in particular cultural contexts, food has a narrative of its own with respect to galvanizing the workforce, engendering affinity and affiliation among peers, and providing a locus around which interpersonal relations might flourish (Plester, 2015).

For many modern organizations, unfortunately, the sharing and consuming of food has largely revolved around what has been dubbed "cake culture" (Royal College of Surgeons, 2016). This refers to organizational customs around food which center on the consumption of mostly hyper-processed foodstuffs that are nutrient-poor, hyper-palatable and rich in refined sugars (a relatively broad term used to refer to natural sugars - those found in fruit in the form of fructose, or dairy products in the form of lactose - that have undergone chemical processing which strips them of their beneficial nutrient properties; Cleave, 2013). While the following images may be over-generalized and do not hold invariantly across all organizational contexts, the sharing of donuts in the break room, the delivery of muffins and cupcakes during extended meeting sessions in the conference room, and slices of cake being distributed in recognition of an employee birthday or meeting of year-end targets, are almost certainly relatable for a number of people working in modern organizations. Sweet foods are often associated with celebration and deserved indulgence, but also serve as stop-gaps (as with the archetypal cupcakes being used for sustenance during an hours-long boardroom conference) and as cheap and convenient pick meups (as when a desk-bound employee unearths a Snickers bar from their desk drawer).

Moreover, the consumption of refined sugar is a characteristic feature of modern dietary patterns, such that many employees are consuming sugar in their private lives in quantities that - particularly when paired with the impacts of cake culture - can be deleterious for their health and wellbeing. As once observed by the nutritionist John Yudkin, "We now eat in two weeks the amount of sugar our ancestors of 200 years ago ate in a whole year." Expanding further on this schism, scholars in nutrition and health science, using an evolutionary discordance framework, have noted that modern sugar consumption levels represent a form of supranormal stimuli consumed at a supraphysiologic level. In other words, there is an evolutionary mismatch between our evolved innate drives to seek out sweet foods as a source of energy (which would have been adaptive under the environmental conditions in which humans evolved, and for which such nutrient sources were incredibly difficult to come by) and the present day consumption of hyper-saccharine, hyper-palatable, nutrient-poor and industrially processed sugars which are widespread, inexpensive and easily accessed (Turner \& Thompson, 2013).

In the following sections, the relationship between the consumption of refined sugar (used interchangeably with simply 'sugar', as it is often colloquially referred to), and its potential effects on employee stress, fatigue and mood will be explored and several theoretical propositions proffered. The 
potential of social contagion in organizations to exacerbate some of these effects will then be outlined, before then turning to the implications the foregoing has for the private and work-life divide and how some of its potentially negative outcomes might be mitigated by HRM practitioners.

\section{SUGAR AND STRESS}

The World Health Organization has deemed stress the "health epidemic of the 21 st century". Stress produces qualitative impacts on employee engagement, morale and productivity, and its quantitative impacts are thought to cost American organizations over $\$ 300$ billion annually in direct costs, with an additional $\$ 190$ billion spent on downstream healthcare costs. Outside of its substantial effects on organizational life specifically, acute stress has been attributed to increasing rates of heart disease, alcoholism and substance abuse, as well as inflating the costs of mental health disorders. While HRM practitioners in many organizations have devoted considerable resources toward reducing workplace stress, its impacts continue to be palpably felt by many in the working population; with over $80 \%$ of American adults reporting some degree of stress in their working lives, and 40-50\% requiring assistance with stress management in the vocational domain. Furthermore, stress can produce deleterious effects on general emotional health in the workplace, with the effects of the relationship between stress and emotionality the basis of a vast extant literature in the organizational and managerial sciences (Siegrist, 2016).

The effects of stress and the consumption of sugar exhibit a bi-directional relationship. As observed in the results of a study recently published in the Journal of Clinical Endocrinology \& Metabolism (Tryon et al., 2015), the brain's ability to mobilize appropriate physiological and behavioral responses to stress is compromised following the consumption of sugar-sweetened foods. 80 percent of those surveyed in this study reported consuming more sweet food when enduring significant psychological stress. Moreover, sugar consumption rates were associated with greater activation in brain regions (namely the left hippocampus) that regulate an individual's sensitivity to stress, as well as substantially reduced bioavailability of cortisol (a hormone released by the body in response to stress). Additional work has demonstrated that blood glucose levels resulting from sugar consumption can produce pronounced pathological effects on stress management capacities. Sudden spikes in blood glucose levels, as occurs with the consumption of refined sugars, can cause the body's adrenal glands to secrete cortisol in a dysregulated manner, rather than contextually regulating its secretion as an appropriate bodily response to stress (Kiecolt-Glaser, 2010). Taken together, it would seem that the upshot for organizations, particularly as it pertains to organizational cake culture, is both that stress can increase one's desire to consume sugar, and that consuming sugar can amplify and intensify one's workplace stress.

\section{Proposition \#la: Individual sugar consumption is positively associated with individual-level organizational stress. \\ Proposition \#1b: Individual-level organizational stress is positively associated with individual sugar consumption.}

\section{SUGAR AND FATIGUE}

While complex and multi-faceted in both its antecedents and causal substructure, fatigue refers generally to a state of inability to perform one's normal activities due to excessive mental and physical exhaustion. It has often been conceptualized as an energy balance problem, with outsized periods of being awake and engaged in physical and/or cognitive exertion paired with inadequate amounts of sleep and other avenues for restoring one's energy levels. Workplace fatigue represents an increasing source of alarm for many modern organizations, with extended work hours, ever-increasing workloads and job demands, and the disruption of healthy circadian rhythms being all too commonplace in many industries. Workplace fatigue is associated with lapses in motivation, alertness, information processing and 
emotional health, and its direct costs to the private and public sectors was once estimated to be more than $\$ 18$ billion per yea (Wachs \& Helge, 2001).

Across the health sciences and several branches of clinical medicine, the relationship between sugar consumption and fatigue is the basis of a wide literature, particularly for applied contexts. The consumption of sugar reliably predicts increases in sleep arousals: lapses in and out of a state of deep sleep which neither produce full wakefulness nor grant the brain the restorative and rejuvenative properties of deep sleep (Cao, Wittert, Taylor, Adams \& Shi, 2016). Sugar consumption can also inhibit the body's ability to deploy melatonin - a hormone partially responsible for regulating sleep and wake cycles in the body. The spiking effect of refined sugar consumption on blood glucose levels can also severely impact the body's faculties for maintaining requisite energy supplies, which can compromise both one's ability to sleep at all, and to sleep sufficiently deeply when they do fall asleep (Ohlmann, O'Sullivan, Berryman \& Lukes, 2009). Furthermore, fatigue and sugar consumption possess a bidirectional relationship relatively analogous to that between stress and sugar consumption. Individuals that are deprived of sufficient rest - at the level of fatigue - are more likely to seek out sugar as a source of easy, accessible energy fulfillment, and those who excessively consume sugar for these energy restoration purposes are more prone to the sort of incidental energy withdrawals (often referred to as "crashes") which are characteristic of chronic fatigue. This has particularly problematic implications for organizations which demand cognitive acuity and focus on the part of their employees. And in the context of cake culture, sugar consumption might potentially be revealed as a culprit in the ever-increasing rates of organizational fatigue.

Proposition 2a: Individual sugar consumption is positively associated with individual-level organizational fatigue.

Proposition 2b: Individual-level organizational fatigue is positively associated with individual sugar consumption.

\section{SUGAR AND MOOD}

A substantial literature in the organizational and managerial sciences has attested to the impact that individual-level mood can have on the wider organizational setting. Particularly in light of the prevalence of teamwork within many industries, there has been an increasing recognition of how and why the interplay of different moods among different people in interpersonal work settings can produce marked effects on organizational outcomes of interest. Indeed, "people are "walking mood inductors," continuously influencing the moods and then the judgments and behaviors of others" and the realities of interpersonal dynamics in team settings "not only...influence people's moods in the group but... influence subsequent group dynamics among group members, both at an individual and group level." (Barsade, 2002:667). Team members are apt to assume the moods of their team leaders, and individuals lower in status within an organization are more likely to behave in a manner congruent with the moods of individuals further up the hierarchy than themselves. Leaders, in particular, are thought to be able to transfer their moods to other members of the organization with great ease given a greater abundance of opportunities to showcase and express their moods to others. Mood contagion (to be explored in more depth in a later section in the context of social contagion), refers to the unconscious, automatic and reflexive transmission of mood between different individuals. Furthermore, unpleasant moods on the part of individual organizational members are more likely to be experienced, onboarded and transmitted by their peers than are positive or uplifting moods (Owens \& Hekman, 2016).

The consumption of sugar has been demonstrated to produce negative effects on mood and mood regulation. The dysregulation of the body's blood glucose management capacities, which often results from sugar consumption, is associated with a reductions in latent mood levels, as well as behavioral instantiations of depressed mood - including an outward sense of pessimism and hopelessness, exhibitions of morbid posturing, being high-strung and irritable, and showing a lack of interest in ordinary aspects of one's everyday life (Benton, 2002). Additionally, sugar consumption can quickly deplete the 
body of essential B vitamins (such as thiamine), which help to regulate mood and a host of other related cognitive functions associated with it (Ottley, 2000). Yet again, an interactive, two way relationship may be at play, comparable to the aforementioned relationships between sugar and stress and sugar and fatigue. Moody people are more likely to consume sugary foods to alleviate the present impacts of their moods, and the consumption of sugary foods will, at least outside of the immediate term, likely lead to the periodic or sustained lowering of mood. Again, when the behavior of the individual is extended to the impact on the collective, particularly in the context of organizational cake culture, this bi-lateral interplay of the two factors in question may have identifiable, and serious, ramifications for organizations and their HRM practices and policies therein.

\section{Proposition 3a: Individual sugar consumption is negatively associated with individual-level organizational mood. \\ Proposition 3b: Negative individual-level organizational mood is positively associated with individual sugar consumption.}

\section{THE MEDIATING EFFECTS OF SOCIAL CONTAGION}

Social contagion (sometimes used interchangeably or complementarily with 'emotional contagion', although they are distinct constructs), is the phenomenon of having aspects of human cognition and affect in one individual trigger or elicit cognitive or affective responses in other individuals around them. Though detailing the technical specificities of the neural and psychological architecture which governs this process is outside the scope of the present paper, social contagion is an adaptive process through which cognitive and emotional mimicry and synchrony is elicited among different individuals as a means of regulating their relationship to one another; something which can happen with or without conscious awareness, but which is unconscious and automatic in the overwhelming majority of instances (Christakis \& Fowler, 2013).

In organizational contexts, social contagion takes on additional dimensions. Oftentimes, work takes place among individuals who are not kin or kith (and as such do not have existing empathic relationships), but who have to function as cohesive units in the midst of variable emotionality among its members, as well as the external pressures of the work environment itself. The collective emotional state of groups has been demonstrated to produce profound impacts upon a range of relevant organizational outcomes, including group rapport and morale and unit performance. Leaders, in particular, have been shown to have significant influence over social contagion, with the showcasing of their moods and emotions tending to trickle down to followers and serving to shape the underlying social milieu (Owens \& Hekman, 2016).

Research from the Max Planck Institute for Cognitive and Brain Sciences has shown that stress can be significantly modulated by social contagion. The mere observation of another person engaged in a stressful activity, or otherwise visibly showing signs of palpable stress, predicts increased cortisol secretion responses in the observer themselves (Engert, Plessow, Miller, Kirschbaum \& Singer, 2014). Chronic stress felt by an individual in isolation is constrained by that individual's own temperament, personality characteristics and general emotional and mood stability. When placed into a group setting, however, individual-level antecedents of stress are themselves influenced by the temperaments, personalities and emotional repertoires of others; serving as a dynamic feedback loop through which chronic stress can be felt more acutely as a function of the collective than it could be within an individual locus. The causal substructure underlying stress responses occurs below the threshold of conscious awareness; an individual is not 'free' to will away the stimulation of their stress regulation systems in response to endogenous stress from another individual. Stress simply takes on a viral form - insidiously transmitting itself from one person to the next via the neural and psychological architectures governing social contagion. 


\section{Proposition 4a: Social contagion mediates the relationship between individual sugar consumption and individual-level organizational stress.}

Regarding fatigue, scholarly work has shown that employee burnout and exhaustion is often at least partially attributable to affective characteristics of the underlying social structure of the organization. Organizations which are emotionally dysfunctional and helmed by leaders with visible negative affect are reliably predictive centers of fatigue on the part of individuals within the organization (Zheng et al., 2015). Moreover, the popularly known phenomenon of contagious yawning underscores the unique interpersonal mimicry and synchrony involved in exhibiting relative levels of fatigue. Just as the visible stress of other individuals can induce stress responses in an observer, so can visible fatigue be similarly received, onboarded and behaviorally copied by an observer (Thompson, 2014). The neural architecture regulating social contagion, and particularly the mirror neuron system which is a major component thereof, is hardwired to empathize with the exhaustion of a fellow conspecific and to generate an outward empathic response in kind.

\section{Proposition 4b: Social contagion mediates the relationship between individual sugar consumption and individual-level organizational fatigue.}

The phenomenon of mood contagion is perhaps the most well-documented of the sub-dimensions of social contagion. It has been empirically demonstrated that listening to or observing another individual's visible mood state is enough to automatically prompt a congruent mood state in the observer/listener. This spontaneous process occurs without the witness having to share the emotional state of the observed participant, and without the witness having any cognizance or recollection of their own mood state being shifted (Bono \& Ilies, 2006). Mood contagion occurs sufficiently underneath the threshold of conscious awareness that people who are assigned a task to occupy themselves in an experimental setting can nevertheless be influenced by the moods of another person; suggesting that mood can be transferred through subtle, subconscious cues that do not tax one's other cognitive resources and faculties. Moreover, effects of this sort have been shown to not be explicable in light of other influences on vicarious feelings such as perspective taking or dispositions towards the other individual, with these factors usually evoking discrete emotional states as opposed to the phenomenologically diffuse effects of differing mood states. While the exact construct definitions that delineate 'mood' and 'emotion' often differ according to the underlying theoretical framework being utilized, Neumann and Strack's (2000) conceptualization of mood posits it as distinct from emotion on account of its absence of noetic (knowledge-based) content. Moods, so conceived, only have experiential representations elicited by sensory input, and are not governed by a conceptual or propositional structure. Thus, in the context of social contagion, mood states simply transfer dynamically from person to person 'unthinkingly', in response to subconscious reception (through the process of exteroception; Rotteeveel \& Phaf, 2007) of another individual's outwardly represented affective contents. As this relates to the relationship between sugar consumption and mood, the net affective effect of consuming sweet food by one individual might translate into a corollary shift in mood state for another individual in their immediate social context.

\section{Proposition 4c: Social contagion mediates the relationship between individual sugar consumption and individual-level organizational mood.}

\section{HRM PRACTITIONER IMPLICATIONS}

\section{Practical Implications}

Chronic stress, fatigue and the effects of negative mood at the collective level are extraordinarily costly to organizations, even when the costs borne are accounted for merely quantitatively - in terms of relinquished profitability and/or productivity. From an HRM perspective, there are additional qualitative effects on employee wellbeing and quality of life - as well as missed opportunities and benefits for these 
employees, which might be difficult to quantify - that are felt by organizations affected by such organizational pathologies (Thomas \& Lankau, 2009). For HRM practitioners, carefully designing policy and implementing practices to mitigate the worst effects of chronic stress, for example, is a complex undertaking that requires an ongoing interface with employees so as to stay suitably attuned to their various needs in this respect. Moreover, and as outlined earlier, HRM practitioners must be mindful of the need to distribute responsibility in a balanced fashion - with the individual and organization both having distinct roles in the management of stress levels within the organization. The emotional health of individuals requires both a supportive work environment geared toward maximizing individual flourishing and wellness, as well as the onus being evenly put on the individual themselves to take ownership of the stress in their lives; including the personal health maintenance dimensions which underlie it (Poelmans, 2003).

As this distribution of onus for HRM practitioners relates to sugar consumption, there appears to be an inherent balance to be struck between an interventionist approach which tactfully provides guidance to an employee with poor dietary habits which might exacerbate their stress levels, fatigue and low mood, and respecting the boundaries spanning their work life and private life. Interestingly, while some research has shown that a majority of employees will tend to adopt a positive posture toward company policies that proactively promote healthy eating habits in the workplace, HRM practitioners themselves are usually disinclined to take such interventionist measures (particularly with respect to an employee's eating habits outside of the workplace) (Quintiliani, Poulsen \& Sorensen, 2010). HRM practitioners tend to see this as transgressing the sanctity of the work-life divide; that one's diet is a private matter outside the organization's purview, and that it falls to the individual to take appropriate steps to eat in accordance with their own health needs.

What's more, the effects of sugar consumption on stress, fatigue and mood are likely not widely known by enough people, HRM practitioners included, to prompt an appetite for proactive intervention. Despite the increasing prevalence of literature admonishing the general public of the dangers of sugar, there is currently little extant material to sculpt HRM policy and practice with respect to the notions that (a) an employee's consumption of sugar might be degrading their cognitive and affective faculties such that it is impacting upon their individual performance and, in turn, the performance of their group or team and the wider organization in which they are embedded and (b) to the degree that sugar can be identified as the source of this degradation, there ought to be a suitably sensitive and scientifically-grounded but interventionist approach that HRM practitioners can use to address the employee and facilitate changes in their dietary habits.

Complicating matters further is the observation that, however otherwise well-motivated and intentioned, companies which have employee wellness initiatives or other organization-wide protocols around health and wellbeing may nonetheless be exposing employees to unhealthy amounts of refined sugar. For example, many organizations have taken to replacing characteristically saccharine foods (such as candy bars and cookies) with so-called "heart-healthy" snack and meal options in workplace vending machines and canteens. Exemplars of such foodstuffs include granola bars and low fat yoghurts; products which, in many cases, have comparable amounts of refined sugars as the "junk food" they were intended to replace (Taubes, 2016).

\section{Ethical Implications}

It might be argued, both by employees and their human resource departments, that the food one chooses to consume will always be a private matter and falls outside the scope of organizational intervention. It is probably reasonable to expect that many people will resist an expectation (much less an intervention) on the part of their organizations that they eat in a manner which maximizes their personal health and wellbeing, however well-motivated that expectation might be and however well-grounded it is in an evidence-based rationale.

And yet it could be conversely argued that HRM practitioners that passively observe the degradation of the cognitive and affective health of employees are behaving unethically in not adopting a more interventionist approach to the matter; particularly with respect to helping correct an eminently alterable 
behavioral pattern, as is the case with sugar consumption. Moreover, HRM practitioners choosing to be so passive might similarly be accused of not behaving in a way that is ethically congruent with the aims of the wider organization; particularly if the proposed mediating effects of social contagion on the relationships between sugar consumption and stress, fatigue and mood are borne out under empirical examination. As alluded to earlier, employees do not simply "switch off" a separate private life mind when they venture out to work. To the degree that what an employee eats in their private life has effects on their cognition and affect, these effects will inevitably be dragged into their work life and exposed to others within the organization. The work-life divide might be a reasonable normative proposition when it comes to what one eats, but it is not a realistic neural, psychological or behavioral proposition.

One might draw an analogy here with tobacco smoking. The relationship between the consumption of tobacco products, particularly through smoking, is well-established and is the basis of a wide literature as well as being a commonly recognized and popularly understood phenomenon among the general public. Given these realities, and the fact that many of the negative health outcomes associated with smoking having implications for organizational outcomes, a number of organizations have increased efforts to minimize or eliminate smoking in the workplace. These measures include outright bans on smoking on company property and proactive health and wellness interventions designed to encourage quitting smoking among affected employees. Some more severe interventions have also included using legal discrimination to guard against hiring smokers as new employees (Gray, 2005). In each case, there is an underlying recognition that consumption of tobacco is both unhealthy for the individual and may in turn be negatively consequential for the organization; dual considerations comprising both ethical and practical dimensions.

If the proposed relationships between sugar consumption, stress, fatigue, mood, and social contagion in organizations were to be borne out empirically, it seems reasonable to at least raise the notion that HRM policy and practice governing sugar consumption - analogous to that governing tobacco consumption - might one day become a practically necessary and ethically obligatory feature of the duty of care mandate in many human resource departments across a number of potentially affected organizations and industries. In times past, smoking would have been as widely considered a private matter not subject to organizational intervention as sugar consumption is at the present moment. Time will tell whether the robustness and weight of evidence underscoring the uniquely deleterious effects of sugar consumption will yield a similar progression in terms of attitudes towards sugar's place in the work-life divide.

\section{FUTURE RESEARCH DIRECTIONS}

The present paper has represented an effort to mount a theoretical case for the proposition that sugar may uniquely produce deleterious effects on individual-level organizational stress, fatigue and mood, and that these effects will be mediated through the process of social contagions, for which organizations are vectors of this phenomenon. This case has been predicated on several converging streams of empirical evidence from a number of complimentary scientific disciplines - both from within the managerial and organizational sciences, and also from fields as diverse as nutrition science, neuroscience and behavioral endocrinology, among others. In terms of the evidence that has been marshaled and presented in this effort, the reader should note that many of the findings invoked are based on observational and/or crosssectional studies, for which causal inferences are difficult to establish (Grimes \& Schulz, 2002).

Indeed, it might be argued that the only truly empirically satisfactory approach to definitively determining a causal relationship between refined sugar consumption and its potential pathological effects on individual and organizational outcomes is to undertake randomized controlled trials (Evans, 2003). Putting such an initiative into practice would almost certainly require randomizing tens of thousands of participants (drawn from multiple, differing organizations and industries) before grouping them into cohorts that are assigned to consume diets with differing levels of sugar consumption. Thereafter, participants would need to be monitored for their stress, fatigue and mood levels in an ongoing fashion, potentially for a number of years afterwards. Such an undertaking would admit of multiple ways for any 
data derived to be significantly compromised: participants may deviate from their assigned diet in their private lives or may not accurately or honestly report their dietary patterns, in addition to other methodological and control considerations, inconsistencies in the external environment across different organizations, and so forth. Moreover, studies of this nature are expensive, time-consuming and resourceintensive.

However, when one considers the implications this paper has proposed for individual wellness in the workplace, and its subsequent potential impacts on organizations generally and HRM in particular, the act of comprehensively establishing causality might not be best viewed as the prime consideration for researchers. While all serious scholars aspire to the best quality of data available, the limitations outlined above, and the urgency with which these phenomena may need to be addressed, may make it such that a more modest research approach will suffice. One may not be able to infer causality from survey-based studies and observational or cross-sectional data, even if multiple studies are carried out across multiple organizations and industries, but such research may nonetheless provide a modicum of evidential support in order to determine the relative scope and dangers of the phenomenon. If, for example, sugar consumption was determined to be strongly correlated with individual-level organizational stress, and that one's place in a social network (determinable through social network analyses) mediated the degree of covariance between these variables, this information might be valuable to HRM practitioners looking to use an evidence-based approach to informing human resource policy and practice. Such information might provide grounds for suitably interventionist initiatives on the part of HRM practitioners, who might otherwise be constrained by a complete absence of meaningful evidence on this front. Researchers might not have satisfied the criteria for causality, but they may have done their part to shine a light on the worst excesses of organizational cake culture, and illuminated some prospects for human resource departments in addressing the worst excesses of it.

Scholars of industry and organizations that are interested in engaging in research in this area would do well to partner with researchers from disciplines across the various sub-disciplines within the health and nutrition sciences to produce interdisciplinary work. The pairing of the former's understanding of the realities of organizational life, and the adverse affects of stress, fatigue and mood on individual-level wellbeing in organization, with the latter's expertise in the mechanisms of metabolism and nutrition, as well as the underlying physical realities of sugar specifically, would likely make for a fruitful, focused avenue of scholarly enterprise. The potential severity of the proposed problem in question would likely demand such a broad degree of scholastic collaboration, particularly given the multi-level considerations across not just individual, team and organization, but across neural and hormonal anatomy, neurological and psychological architecture, and cognitive, affective and behavioral output. Such collaborative efforts would also represent a timely and potentially greatly consequential addition to an ever-expanding canon of published work that has squarely centered on the dangers of sugar in modern human dietary patterns; providing a lens for this area of examination that has considerable applicability and relevance for a great number of potentially affected people across today's working environments.

\section{REFERENCES}

Barsade, S. G. (2002). The ripple effect: Emotional contagion and its influence on group behavior. Administrative Science Quarterly, 47(4), 644-675.

Bono, J. E., \& Ilies, R. (2006). Charisma, positive emotions and mood contagion. The Leadership Quarterly, 17(4), 317-334.

Cao, Y., Wittert, G., Taylor, A. W., Adams, R., \& Shi, Z. (2016). Associations between macronutrient intake and obstructive sleep apnoea: Results from a cohort of community dwelling Australian men. Nutrients, 8(4), 207.

Carrera-Bastos, P., Fontes-Villalba, M., O'Keefe, J. H., Lindeberg, S., \& Cordain, L. (2011). The western diet and lifestyle and diseases of civilization. Research Reports in Clinical Cardiology, 2, 15-35.

Christakis, N. A., \& Fowler, J. H. (2013). Social contagion theory: examining dynamic social networks and human behavior. Statistics in medicine, 32(4), 556-577. 
Cleave, T. L. (2013). The saccharine disease: conditions caused by the taking of refined carbohydrates, such as sugar and white flour. Amsterdam: Elsevier.

Engert, V., Plessow, F., Miller, R., Kirschbaum, C., \& Singer, T. (2014). Cortisol increase in empathic stress is modulated by emotional closeness and observation modality. Psychoneuroendocrinology, 45, 192-201.

Ernst Kossek, E., Lewis, S., \& Hammer, L. B. (2010). Work-life initiatives and organizational change: Overcoming mixed messages to move from the margin to the mainstream. human relations, 63(1), 3-19.

Evans, D. (2003). Hierarchy of evidence: a framework for ranking evidence evaluating healthcare interventions. Journal of clinical nursing, 12(1), 77-84.

Gray, N. J. (2005). The case for smoker-free workplaces. Tobacco Control, 14(2), 143-144.

Grimes, D. A., \& Schulz, K. F. (2002). Bias and causal associations in observational research. The Lancet, 359(9302), 248-252.

Johnson, R. J., Sánchez-Lozada, L. G., Andrews, P., \& Lanaspa, M. A. (2017). Perspective: A historical and scientific perspective of sugar and its relation with obesity and diabetes. Advances in Nutrition: An International Review Journal, 8(3), 412-422.

Kiecolt-Glaser, J. K. (2010). Stress, food, and inflammation: psychoneuroimmunology and nutrition at the cutting edge. Psychosomatic Medicine, 72(4), 365.

Kramar, R. (2014). Beyond strategic human resource management: is sustainable human resource management the next approach?. The International Journal of Human Resource Management, 25(8), 1069-1089.

Mansur, R. B., Brietzke, E., \& McIntyre, R. S. (2015). Is there a "metabolic-mood syndrome"? A review of the relationship between obesity and mood disorders. Neuroscience \& Biobehavioral Reviews, 52, 89-104.

Maslach, C., Leiter, M. P., \& Jackson, S. E. (2012). Making a significant difference with burnout interventions: Researcher and practitioner collaboration. Journal of Organizational Behavior, 33(2), 296-300.

Neumann, R., \& Strack, F. (2000). " Mood contagion": the automatic transfer of mood between persons. Journal of personality and social psychology, 79(2), 211.

Ohlmann, K. K., O'sullivan, M. I., Berryman, P., \& Lukes, E. (2009). The costs of short sleep. AAOHN journal, 57(9), 381-387.

Ottley, C. (2000). Food and mood. Mental Health Practice, 4(4), 32-39.

Owens, B. P., \& Hekman, D. R. (2016). How does leader humility influence team performance? Exploring the mechanisms of contagion and collective promotion focus. Academy of Management Journal, 59(3), 1088-1111.

Plester, B. (2015). Ingesting the organization: The embodiment of organizational food rituals. Culture and Organization, 21(3), 251-268.

Poelmans, S. (2003). The multi-level 'fit'model of work and family. International Journal of Cross Cultural Management, 3(3), 275-288.

Quintiliani, L., Poulsen, S., \& Sorensen, G. (2010). Healthy eating strategies in the workplace. International journal of workplace health management, 3(3), 182-196.

Rotteveel, M., \& Phaf, R. H. (2007). Mere exposure in reverse: Mood and motion modulate memory bias. Cognition and Emotion, 21(6), 1323-1346.

Royal College of Surgeons (2016). Workplace 'cake culture' blamed for rise in obesity. Retrieved from https://www.rcseng.ac.uk/error/not-found/?item $=\% 2$ fnews $\% 2$ f 2018 cake-culture 2019 -puttingoffice-workers2019-health-at-risk-warns-dentalleader\%2f\&user $=$ rcseng $\% 5 c$ Anonymous\&site=website\#.V2zd7JMrJTY

Siegrist, J. (2016). Stress in the workplace. In Cockerham (Ed). The New Blackwell Companion to Medical Sociology, Hoboken, NJ: Wiley 268-283.

Taubes, G. (2016). The case against sugar. New York: Knopf. 
Thomas, C. H., \& Lankau, M. J. (2009). Preventing burnout: The effects of LMX and mentoring on socialization, role stress, and burnout. Human Resource Management, 48(3), 417-432.

Thompson, S. B. (2014). Yawning, fatigue, and cortisol: expanding the Thompson Cortisol Hypothesis. Medical hypotheses, 83(4), 494-496.

Tryon, M. S., Stanhope, K. L., Epel, E. S., Mason, A. E., Brown, R., Medici, V.,. \& Laugero, K. D. (2015). Excessive sugar consumption may be a difficult habit to break: a view from the brain and body. The Journal of Clinical Endocrinology \& Metabolism, 100(6), 2239-2247.

Turner, B. L., \& Thompson, A. L. (2013). Beyond the Paleolithic prescription: incorporating diversity and flexibility in the study of human diet evolution. Nutrition reviews, 71(8), 501-510.

Wachs, J. E., \& Helge, D. (2001). Turning workplace anger and anxiety into peak performance: Strategies for enhancing employee health and productivity. Aaohn Journal, 49(8), 399-408.

World Health Organization (2015). Sugars intake for adults and children. Retrieved from http://www.who.int/nutrition/publications/guidelines/sugars intake/en/

Zheng, D., Witt, L. A., Waite, E., David, E. M., van Driel, M., McDonald, D. P.\& Crepeau, L. J. (2015). Effects of ethical leadership on emotional exhaustion in high moral intensity situations. The Leadership Quarterly, 26(5), 732-748. 